\title{
Traditional Chinese medicine approach to hypothyroidism
}

Volume 5 Issue I - 2017

Damir Malikov

Acupuncturist \& Herbalist in Los Angeles, USA

Correspondence: Damir Malikov, Acupuncturist \& Herbalist in Los Angeles, USA, Tel (323)-422-6563,

Emailinfo@drmalikov.com

Received: August 22, 2016 | Published: January 03, 2017

deiodinase expression, to modify the effects of T4 and T3; or thyroid gland auto-regulation in response to iodine availability.

\section{Hypothyroidism}

Hypothyroidism is defined as inadequate peripheral tissues stimulation by the thyroid hormone. This deficiency is caused by either failure in adequate thyroid hormone production or reduction in tissue sensitivity to the thyroid hormone stimulation. The decreased thyroidal secretion of thyroid hormone, due to the factors affecting the thyroid gland itself is called "primary" hypothyroidism; decrease in serum concentrations of thyroid hormone caused by an inadequate TSH secretion, due to factors directly affecting the anterior pituitary gland is called "secondary" hypothyroidism; the failure of TRH release, due to the factors affecting hypothalamus, will cause an indirect decrease in the secretion of thyroid hormones called "tertiary" hypothyroidism. The secondary and tertiary hypothyroidism is often referred to as "central hypothyroidism."

Hypothyroidism with onset in adulthood causes a generalized slowing down of the organism, with deposition of glycosaminoglycans in intracellular spaces, particularly in skin and muscle, producing the clinical picture of myxedema. Hypothyroidism is graded on a scale ranging from Grade 1-mild cases in which the individual hardly notices signs and symptoms thyroid hormone deficiency, to Grade 3 -severe cases in which severe unregulated thyroid insufficiency may cause myxedema coma and death. Most symptoms of hypothyroidism in adults are largely reversible with therapy.

\section{Traditional Chinese medicine approach to hypothyroidism}

Traditional Chinese medicine has for several thousand years been the treatment of choice for over a quarter of the world's population for its ailments, infections, and other health problems. Today, Traditional Chinese Medicine (TCM) is practiced in China alongside with Conventional Western Medicine. Most hospitals include departments 
of complementary medicine which offer acupuncture treatment and herbal medicine. The acupuncture in United States is most often associated with pain management and builds on a 'conventional' interpretation of how the body functions.

Traditional Chinese medicine is every bit as logical and systematic as Conventional Western medicine and is based on a different understanding of reality. TCM has for centuries made use of the same basic concepts like Qi and the balance of Yang and Yin to explain various universal phenomena.

Although it is difficult to guess when humans first started using herbs as medicine; the Traditional Chinese Medicine, as a form of medicine practice today, dates 4000-6000years back in time. While acupuncture is the best known modality of TCM in the West, this medicine is by no means is just limited to acupuncture. Chinese medicine makes great use other therapeutic modalities like herbal remedies, acupuncture, auricular acupuncture, cupping, Tui Na; as well as Tai Ji and Qigong as forms of physical therapy.

\section{Differences between TCM and conventional western medicine}

In Conventional Western schools of medicine body is studied as a sum of its parts, and every organ system of the body is judged and treated separately. Hospitals are specialized by different departments associated with the diagnoses and treatment of different organ systems: one for the cardiovascular patients; another for the endocrinology; yet another for surgery. Traditional Chinese Medicine is holistic, where the body is treated as a 'whole,' and every part of the body is seen as a reflection of the wider context. A particular body part is referred to only in relation to the whole body, and the body must also be seen as intimately connected with the spirit and the surrounding environment in which the patient lives before a doctor of TCM can understand how an illness has arisen and how it should be addressed.

\section{Thyroid deficiency in TCM}

Thyroid disorders are some of the most common clinically encountered endocrine disorders worldwide. Until the resent discovery of the more accurate understanding of the thyroid anatomy and its role in human physiology as a major endocrine organ, as well as the introduction of new imaging techniques and serum thyroid function tests into the modern medical practice; the ancient physicians were limited to few general symptoms and basic physical evaluation to diagnose disorders related to the thyroid gland. The diagnosis in the Traditional Chinese Medicine (TCM) pattern was limited to detection of common signs and symptoms often seen in hypothyroidism and palpation of neck nodules in the area of the thyroid gland. In modern TCM practice objective measurements, such as thyroid hormone levels, TSH values and the presence of autoreactive immunoglobulins, can clarify the nature of the disease and give a clue to a various herbal therapeutic approaches that might be effectively applied in the treatment of hypothyroidism. Even though, the clinical management of hyperthyroidism cases has been of a greater interest in the Traditional Chinese Medicine field, the hypothyroidism is no less prevalent in the modern day TCM practice. The treatments may vary from one physician to the next, but there are certain consistent features which will be discussed mainly in regards to management of patients with hypothyroidism. The clinical manifestations of hypothyroidism are associated with consumptive deficiency, edema, goiter and other signs and symptoms according to the TCM differentiations and pathogenesis.

\section{Etiology and pathogenesis}

The hypothyroidism, as described in Traditional Chinese Medicine texts, is classified mainly as the deficiency disorder. The diagnosis must be differentiated and often varies from case to case depending on the individual patient's clinical presentation. It is important to understand that hypothyroidism is the condition arising due to the chronic deficiency of one or more vital substances of the body: Qi, Blood, Yang, Yin and Essence. This is a systemic disorder which may start with general Qi deficiency and affect all organ systems over the long period of disease progression. The Spleen, Kidney and Heart are three primary organs involved in the pathogenesis of hypothyroidism. The Lung and Liver are also affected in an indirect manner. Manifestations of various deficiencies are diverse and can affect a wide variety of physiological processes. The precipitating causes of hypothyroidism include constitutional factors, diet, overexertion, emotional stress, trauma, chronic debilitating illness, as well as the side effects of medical treatment. Patterns of deficiency will be differentiated by aspect of underlying deficiency of Yin, Yang, Qi or Blood in regards to the deficient organ, or a combination of organs, involved in pathogenesis: Spleen, Kidneys, and Heart.

The clinical manifestations of the hypothyroidism emerge gradually as the result of slowing metabolism and depletion of body's Qi which develops into the Yang deficiency. As the Yang qi deficiency becomes more severe it begins to fail in its functions of warming, motivation, and transformation, resulting in the decline of multiple organ functions and classical set of symptoms often seen in patients with clinical hypothyroidism. The initial stage of the disease is equivalent to the subclinical hypothyroidism, and marked mainly by the Spleen Qi deficiency and is often associated with its symptoms. As the disease remains untreated the Ming men fire begins to fail to compensate the Spleen Yang deficiency. As the result more, Kidney Yang deficiency symptoms arise and the Spleen Yang deficiency symptoms worsen, marking the stage of clinical hypothyroidism. Thus, Yang deficient Kidney will fail to warm the Spleen Yang Qi, resulting in severe Yang depletion in both organs. When Spleen Qi is deficient the generation of the Source Qi and Blood is inadequate, resulting in Kidney Essence depletion. It may be years before the Kidney Ming men fire begins to fail to warm the Heart Yang, which will lead to Heart Yang deficiency. Untreated Yang deficiency will result in the failure of fluids metabolism and will cause in the excess water to overflow towards the Heart and lead to cardiac dysfunction and associated symptoms commonly seen in patients with advanced stage of overt hypothyroidism. Even though, it is extremely rare to encounter patients with severe hypothyroidism in the developed countries due to proper diet, improved screening, timely diagnosis and treatment of the disease, the hypothyroid patients who remain untreated may have about ten to fifteen years before their basic body functions will start failing.1 Thus, the severe Yang deficiency will inevitably result in the complete depletion and devastation of the Ming men fire which will manifest as a number of life threatening functional failures. Note that the Kidney Yang and Ming men fire refers to the fire at the gate of vitality and will be used interchangeably in this report.

\section{Factors contributing to the disease}

Common contributing factors of the thyroid deficiency include: improper diet, iatrogenic and constitutional factors and overexertion.

\section{Improper diet}

Irregular dietary intake, excessive consumptions of raw and cold 
foods, like bok choy, will injure Spleen Yang Qi ${ }^{4}$. Chronic iodine and selenium deficiency will also contribute to the rise of hypothyroid state, previously believed to be the 'evil Qi' and sand in the water of springs and rivers, especially in the mountainous regions.

\section{latrogenic factors}

Long term use or unusually high doses of bitter and cold herbs, emetics, and purgatives, have direct impact on Spleen and Stomach Yang Qi, and may impair their functions of transportation and transformation of food and fluids.

\section{Constitutional factors}

Constitutional factors include congenital deficiency and prenatal malnourishment, both of which may play role in development of thyroid deficiency. In Women the depletion of Essence and Blood from repeated births will directly drain the Kidney, Liver and Spleen.

\section{Overexertion}

Overtaxation and exhaustion of Kidney Yang by physical, mental or sexual activities is also one of contributing factors of hypothyroidism. It is apparent that hypothyroidism is the result of both Qi and Blood deficiency which gradually develops into the Yang and eventually Yin deficiency.

\section{Clinical picture}

Hypothyroidism, like most other endocrine disorders, develops gradually over the period of several years and even decades. Many symptoms can erroneously be attributed to general environmental factors and natural aging. Patients often seek medical help when the symptoms begin to interfere with their daily activities.

As the disease progresses the symptoms will worsen and more severe symptoms may manifest. Even though, the symptoms of hypothyroidism come in a great variety they all arise from the core pathogenesis of the deficiency in Yang Qi that is failing to warm, defend, consolidate and transform.

\section{Fatigue and weakness}

This is the earliest of symptoms of subclinical hypothyroidism while the thyroid deficiency may not be apparent. It is very difficult to diagnose the subclinical hypothyroidism this early without the serum TSH analysis, and even then the western physician will often postpone the treatment until more severe symptoms appear. In TCM fatigue and weakness, especially on exertion, is associated with general Qi deficiency and suggests the involvement of Spleen in early pathogenesis of the disease. The 'leg Qi' and atrophy disorder may arise as the result of either dampness or chronic Spleen deficiency. This pattern is discussed in the Essential Questions which says "With exposure to dampness over a period of time, the muscles and flesh will be invaded; this will lead to insensitivity and flesh atrophy" and "When there is illness in the Spleen it fails to transport body fluids for the Stomach. The four limbs do not receive the nourishment of water and grain and therefore become weak. There is no free flow through the vessel-pathways and there is no qi to engender the sinews, bones and muscles which therefore cannot function ${ }^{5}$. Thus, Spleen Qi deficiency will also lead to development of such symptoms as decreased appetite, muscle weakness, delayed reflex time, anorexia, distention and bloating especially in the latter stage of the disease. Chronic Spleen deficiency will cause blood deficiency and anemia, and also result in Qi stagnation leading to muscle spasms, cramps, myalgia and even ataxia.

\section{Cold intolerance}

It is the most definitive symptom of the thyroid deficiency. Qi is yang and possesses a warming quality. Qi maintains and regulates the body temperature. Aversion to cold is classically associated with the deficiency in Yang qi, which fails to adequately warm the body. This symptom may often be missed in the early stages of the disease because the patient usually starts by compensating behavior: avoiding the cold by dressing warmer and increasing the room temperature. As the body's Yang qi weakens the internal cold starts accumulating, this phenomenon is also known as Yang deficiency generating internal cold, which may lead to slowing or even freezing of physiological processes. Sweat is generated via the transformation of fluids by the Spleen and Kidney Yang qi. The deficiency in Yang qi consequently results in hypohidrosis (partial lack of sweating) and consequently myxedema (internal fluid accumulation) due to the failure of Qi to open the pores and regulate the fluid metabolism or cold pathogen closing the pores shut. Usually patients will complain of the general Qi deficiency symptoms like pallor, fatigue, lethargy and muscle weakness, edema, deficient constipation and indigestion, depression, impaired memory and slow speech. Tongue and pulse will reflect the underlying Qi deficiency and is often pale, swollen with teethmarks, with thin slippery coating. The pulse will be deep, forceless, and slow but regular, suggesting Yang deficient cold.

It is important to differentiate the aversion to cold and the lack of sweat in hypothyroidism to Wind-Cold invasion. Wind-Cold is an external pathogen invading the body and resulting in an 'excess' condition which will not be alleviated by warm blanket or more clothes.

\section{Constipation}

Constipation is another major symptom of hypothyroidism. Diminishing Yang will eventually result in internal deficient cold accumulation in the Large intestine causing reduced bowel motility and obstruction. Other gastrointestinal symptoms may include cold epigastric or abdominal pain and spasms, which are relieved by warmth and pressure, as well as poor appetite and indigestion, accompanied by deep slow pulse.

\section{Edema}

Kidney stores Ming men fire, which helps to metabolize fluids. In hypothyroidism, when Kidney Yang is deficient, the water circulation in the body is disrupted, leading to pathogenic derivatives that are cold and Yin in nature, resulting in systemic edema.5 The edema is nonpitting and is often seen on the face, the skin may be thick and difficult to pinch. The later stage of hypothyroidism is marked by peritibial edema which may be pitting. Fluids may also accumulate in the joints, causing swelling and joint pain and even carpal tunnel syndrome. As the disease advances towards the later stage, water often overflows the heart and the lung resulting in the pericardial and pleural effusions. Consequently, angina, chest congestions, palpitations, slow pulse and slow breathing, dyspnea are common in myxedematous patients; as well as the pleural effusions resulting in chronic respiratory infections and eventually leading to myxedema crisis and coma. In TCM, myxedema coma is also perceived as accumulation of turbid, cold, Yin pathogens in the pericardium. ${ }^{6}$

\section{Dry and flaky skin, thinning of hair or loss of hair}

Spleen and Kidney Yang deficiency result in Blood and Essence depletion. Spleen Yang qi is responsible for generating Qi, Blood, and Essence. The deficiency in Spleen Yang qi in hypothyroidism results 
in the failure of the Spleen to distribute nutrients to the body, resulting in Blood and Qi deficiency causing dry and flaky skin. The kidney manifests in the hair, thus as the Kidney Yang and Essence is depleted the hair becomes dull, thin, and more scares.

\section{Irregular menstruation and menorrhagia}

Hypothyroidism is much more common in women than men, and often disturbs women's menstruation. Qi is one manifestation of Essence. The patency of Liver Qi is important to help Spleen Qi function well. Severe Qi and Blood deficiency in hypothyroid women often disrupts the circulation of Liver Qi and Chong Mai which are responsible for smooth menstrual cycles and release of the menstrual blood, and results in menses which are often irregular and sometimes anovulatory, causing infertility. Pale menorrhagia is also common. In hypothyroid men chronic Kidney Yang Qi and consequent Essence deficiency may manifest as impotence and infertility.

\section{Other signs and symptoms}

Many patient with the progressive hypothyroidism present with Heart and Kidney deficiency leading to Shen disturbance manifesting as depression, reduced attention span, memory deficit, slowed reaction, husky voice and vertigo.

\section{Differential diagnosis}

The first step in diagnosis of hypothyroid condition according to TCM is to determine the nature of deficiency in the patient's energy field that gives rise to the presented signs and symptoms. Second step is to differentiate, the suspected diagnosis to create the most efficient treatment plan. The disorder may then be treated with herbs and acupuncture, or even cupping, Tai Ji, Qi Gong or Tui Na if so desired. An experienced physician will seek a 'pattern in the fabric' of the condition and choose a treatment that favorably influences that pattern. One may begin by asking the patient about the symptoms, checking the signs, including pulse and tongue diagnosis. For example there may be detailed questions on possible perspiration, feelings of warmth or cold, on the patient's bowel movement and digestion, evacuation of urine, and in the case of female patients, fertility and menstruation, even if the patient's illness might be seen and having no connection with such matters. The key is to find a pattern in the balance between Yin and Yang and of the flow of Qi in the body, using the information gathered in order to gain an understanding of how the various organs are working together, and what went wrong. An experienced physician, may also perform an examination of the ear, by visual inspection and palpation; and any changes in color, shape and possibly sore points or points with a changed electrical resistance will be noted.

\section{Major TCM patterns of hypothyroidism Spleen QI Deficiency}

The early stage of subclinical hypothyroidism is associated with Spleen Qi deficiency, which if untreated will develop into more serious Spleen Yang deficiency, which will further affect the function of all remaining organs. General symptoms of Qi deficiency include lethargy and fatigue, which are precipitated by physical activity and improve with rest, which are key symptoms associated with subclinical hypothyroidism. Other common symptoms of Spleen Qi deficiency are shortness of breath (on exertion), concise, slow and occasionally slurred speech, husky voice, dusky complexion, reduced appetite, loose stools, and a weak pulse. a. Herbal treatment: Si Jun Zi Tang or 'Four-Gentleman decoction' formula is recommended in the treatment of subliminally hypothyroid patients with Spleen Qi deficiency. This formula tonifies the Spleen, augments the Qi and raises and lifts the clear Yang Qi, promoting the circulation in the Middle jiao, and lifts the sunken.

\section{Spleen yang deficiency}

Since, the Spleen and Stomach control the transformation and transportation of food and nutrients they play an essential role in regulating the ascension and discretion of the Qi. In clinical hypothyroidism, when the Yang Qi of the Middle jiao is weak, the patient may suffer an invasion of external cold, which may hinder the normal Qi flow and result in epigastric and abdominal distention or even pain. Spleen Yang deficiency is characterized by intolerance and aversion to cold, clod extremities, poor appetite, pallor, fatigue (cold type), epigastric and abdominal cold pain relieved by warmth and pressure, constipation, distention and excessive thin white leucorrhea in women. The tongue is usually flabby and pale with teethmarks and with white and slippery coating. The pulse is deep, slow and forceless or faint in severe thyroid deficiency.

Herbal treatment: The treatment principle for the Middle jiao Yang deficiency would be to warm the Yang, disperse cold, augment the Qi and to strengthen the Spleen. Thus, most herbs used to treat this condition are warming and acrid, and somewhat dry in nature. Gan Jiang (zingiberis rz) is one of such herbs used in the treatment of most patterns of hypothyroidism. Other frequently used herbs are Fu $\mathrm{Zi}$ (aconiti rx lateralis) and Rou Gui (cinnamomi cortex), which can be combined with herbs that strengthen the Spleen and augment the Qi, like Huang Qi (astragali rx), Ren Shen (ginseng rx), and Bai Zhu (atractylodis macrocephalae $\mathrm{rz})^{7}$

\section{Qi and blood deficiency}

It is said that the 'Qi is the commander of Blood, and Blood is the mother of Qi. ${ }^{8}$ Qi and Blood are mutually connected, 'when Qi moves the Blood follows,' Blood in turn nourishes the Qi, and thus tonifying one will have the indirect effect on the other. Low Red Blood cell count and hypoxia are clinical manifestations of chronic hypothyroidism associated with Qi and Blood deficiency which is marked by fatigue, weakness, slowness, palpitations, sallow complexion, amenorrhea, pale tongue and choppy pulse.

Menorrhagia is a common presentation of clinical hypothyroidism, occasionally accompanied with recurrent pain, or restless fetus disorder. From the Traditional Chinese medicine perspective the pathogenesis is associated mainly with the complex of functional system of the Liver, Chong Mai, and Uterus, known as the 'sea of Blood. ${ }^{8}$ If the Liver Blood is deficient, it is unable to rise and nourish the head, resulting in dizziness, vertigo, and impaired vision. When the Sea of Blood is empty the menstrual cycle is typically irregular with a scanty flow; however, "when the Blood becomes disordered, menorrhagia may also occur.' The tongue is usually pale and pulse is thin and wiry; choppy pulse may be felt in the condition of Blood stasis.

\section{Herbal treatment}

Use modified Shi Quan Da Bu Tang (All-Inclusive Great Tonifying Decoction) to warm and tonify Qi and Yang and stimulate the production and transformation of Blood.

\section{Spleen and kidney yang deficiency}

The difference between the deficient cold and external excess 
cold is that; cold due to Yang deficiency, may be alleviated by simply wearing more clothing, where as cold sensation and chills associated with an exterior pathogen cannot, even by increasing the room temperature. The symptoms like lassitude, lethargy and withdrawal into oneself, aversion to cold, cold extremities, soreness and weakness of the lower back and knees, a flabby pale tongue with white moist coating, and deep slow and forceless pulse (especially at the right proximal position) seen in severe clinical hypothyroidism are some of the common manifestations of Spleen and Kidney Yang deficiency.

\section{Herbal treatment}

You Gui Wan can be used to treat this Yang deficiency pattern of progressive clinical hypothyroidism, and its primarily focus is tonification of the Kidney Yang, because the Kidneys' Ming men fire is the source Yang at the gate of vitality.

\section{Heart and kidney yang deficiency}

Kidney is the root of the body's Yang Qi, which supports the generative and transforming processes of all other organs, chronic Kidney Yang deficiency will result in Spleen Yang deficiency. Water and dampness accumulation, in the late stages of hypothyroidism, will impair the Yang Qi from ascending the clear and descending the turbid, which may obstruct the sensory orifices. Thus, overtly hypothyroid patients often complain of impaired hearing, heavy sensation in the head, dizziness ranging from lightheadedness and positional vertigo to severe dizziness even when lying down. The patient also feels weakness and instability in posture which sometimes requires a conscious effort to maintain.

When pathogenic water ascends and overflows the Heart, it causes palpitations and when it overflows the Lungs and disrupts the Qi, it causes cough and dyspnea. Clinical manifestation of Heart and Kidney Yang deficiency are marked by palpitations, chest congestion, angina, lassitude, lethargy, aversion to cold, cold extremities, absence of sweating, soreness and weakness of the lower back and knees, hoarse voice, slow response to stimuli, and severe systemic myxedema. The pale or dark swollen or flabby tongue with tooth marks, the white, slippery tongue coating, and the deep, slow, thin, forceless, irregular pulse are signs of Yang deficiency with internal retention of pathogenic water and dampness.

\section{Herbal treatment}

A variation of Zhi Gan Cao Tang (Prepared Licorice Decoction) in combination with Zhen $\mathrm{Wu}$ Tang (True Warrior Decoction) can be used to warm and unblock the flow of the Heart Yang, warm the Kidney and promote urination in overtly hypothyroid patients.

\section{Depletion and devastation of yang}

If the condition of debilitated Yang and exuberant Yin with cold in the interior is left untreated, it will eventually lead to Yin repelling Yang, and result in 'floating Yang' syndrome. This pattern of devastated Yang Qi due to its chronic depletion is associated with myxedema crisis, cold inversion pattern, and characterized by pale and dusky complexion, dizziness and blurred vision, icy-cold extremities, shallow and weak respiration, flaccid and weak muscles, somnolence, or even myxedema coma; dusky pale tongue and minute almost imperceptible pulse. In severe cases the Yang will float and the patient may break into a strong profuse sweat.

\section{Herbal treatment}

Use Si Ni Jia Ren Shen Tang (Frigid Extremities Decoction with Ginseng) to warm and rescues the devastated Yang from collapse, reverse peripheral frigidity, and to warm the Middle jiao to transform turbidity.

\section{Treatment of hypothyroidism with acupuncture $\&$ moxibustion}

Hypothyroid patients are often deficient in Yang and Qi, long needle retention or strong acupuncture stimulation may drain Qi, tonification techniques are highly advised and the use moxibustion is encouraged where applicable. It is also important to keep in mind, that as a general rule, Back-shu points tonify the Zang organs and regulate the $\mathrm{Fu}$ organs, while Front-mu points sedate the $\mathrm{Fu}$ and regulate the Zang.

Dan Xi Xin Fa (Dan -xi's Heart Methods) recommends: "For great disease due to vacuity desertion which is Yin vacuity, use Ai Ye (artemisiae argyi folium) moxa at the Dan Tian (Ren 6-4) in order to supplement Yang. When Yang grows, Yin is lengthened also grows. ${ }^{10}$,

\section{Treatment protocols}

The key points for the treatment of signs symptoms and the root cause of clinical hypothyroidism will be discussed in a great detail in this section. The summary of these points is presented in Table 1.

Table I Nine key points for treatment of hypothyroidism

$\begin{array}{llll}\text { Du4 (Ming Men) } & \text { St36* (Zu San Li) } & \text { UBI7 (Ge Shu) } & \text { Li4* (He Gu) } \\ \text { Ren4 (Guan Yuan) } & \text { Sp6* (San Yin Jiao) } & \text { UB23 (Shen Shu) } & \text { P6 (Nei Guan) } \\ \text { Ren6 (Qi Hai) } & \text { (Nei (Shen }\end{array}$

Ren6 (Qi Hai) Sp6* (San Yin Jiao) UB23 (Shen Shu) P6 (Nei Guan)

Several treatment plans are presented below. The plans are similar in construction, and can be used independently, depending on which modality a practitioner prefers, or which therapy the patient would best tolerate.

\section{Supplementary points for various patterns}

For Spleen Qi Deficiency and Spleen Yang Deficiency (Subclinical and Clinical Hypothyroidism) add Ren12 (Zhong Wan).

In Qi and Blood Deficiency (Chronic Clinical Hypothyroidism with cognitive and psychoemotional deficiencies) add Du20 (Bai Hui). ${ }^{11}$

For Spleen and Kidney Yang Deficiency (Chronic Clinical Hypothyroidism) with stifling sensation chest and speech impairment add Ren12 (Zhong Wan) and Ren17 (Shan Zhong).

In Heart and Kidney Yang Deficiency (Overt Hypothyroidism) use Ren17 (Shan Zhong) in addition to a 'Guest-Host' combination of Sp3 (Tai Bai)-Yuan-host and St40 (Feng Long)-Luo-guest to resolve Phlegm due to Spleen deficiency to treat pleural and pericardial effusions, coronary artery disease due to hyperlipidemia and QiPhlegm goiter.

In Depletion and Devastation or Collapse of Yang Qi (Myxedema Crisis) add Du20 (Bai Hui) with Ren17 (Shan Zhong).

For Abandonment Disorder (Myxedema Coma) add Du20 (Bai Hui) and needle Du26 (Ren Zhong), to resuscitate the patient from acute syncope. Most hypothyroid patients became comatose when their respiration fails, thus it is important to keep patient conscious until the paramedics arrive.

\section{Auricular acupuncture}

Few of the points that can be added to acupuncture treatment protocols for hypothyroid patterns: 
General points for hypothyroidism use 'Endocrine', 'Thyroid', 'Neurogate', 'Subcortex.'

\section{Conclusion and review}

It is clear, that the thyroid is one of the most important endocrine glands that produce hormones to regulate metabolism of the whole body including: brain, respiration, cardiovascular and nervous system functions, body temperature, muscle strength, skin dryness, menstrual cycles, weight, energy and water metabolism.

Despite great progress made in medical technologies, all practitioners should strive to make physical and psychological contact with patients, to acquire more complete understanding of functional disorders, without relying on conventional techniques along. This diagnostic approach, however, requires the sufficient medical knowledge to interpret and experience to correlate such information.

According to records of accumulated knowledge generated by the sages of Traditional Chinese Medicine over several millennia, through close observation and experience of treatment of patterns closely associated with hypothyroidism, it is understood that this is a condition of true deficiency in Yang Qi of the body. The etiology of hypothyroidism is rooted in improper diet, overexertion, or environmental factors. Several patterns were discussed to reflect the pathogenesis of the thyroid deficiency and explain the mechanism of the progression of the disease from Spleen Qi deficiency, to development of Spleen, Kidney and eventually Heart Yang deficiency, and further involvement of the Lungs and Liver, and eventually exhaustion and collapse of Yang, finally resulting in coma as the condition continues to worsen.

In general, the Traditional Chinese Medicine management of overtly hypothyroid patients in the modern practice will often be complimentary to standard Conventional Western hormone replacement therapy. When treating a hypothyroid patient, it is important to always address the root cause of the thyroid deficiency. The herbal and acupuncture therapy can often be used in conjunction with the hormone replacement therapy to achieve greater results and to subdue the adverse effects of levothyroxine. It is essential to remember, however, that all herbal preparations, including tonifying formulas have very specific indications and associated effects, and should never be used thoughtlessly.

The practitioner must be able to most efficiently help to promote functional balance and revitalize the patient.

Exercise and proper nutrition are also helpful modalities to support the treatment of hyperthyroidism. As always, it is important to always provide a patient, caring treatment, and to offer behavioral recommendations about lifestyle factors that may benefit the patients or consume essence and aggravate the deficiency in the body.
The thyroid tissue, even after a partial thyroidectomy, iodine irradiation, autoimmune or inflammatory destruction has a potential to revive and resume the production of adequate amounts of thyroid hormones. Human body poses mechanisms of self-restoration, and a caring physician should attempt to help the patient by restoring the thyroid glands function, to help the patient live a complete life full of vitality.

\section{Acknowledgments}

None.

\section{Conflicts of interest}

Author declares there are no conflicts of interest.

\section{Funding}

None.

\section{References}

1. Ingbar SH, Woeber KA. Harison's Principles of Internal Medicine. $10^{\text {th }}$ edn. New York, USA: Diseases of the Thyroid; 1983. pp. 111

2. Greenspan FS, Gardner DG. Basic \& Clinical Endocrinology. $7^{\text {th }}$ edn. USA: McGraw-Hill Medical; 2004. pp. 217-291.

3. Patton PT, Thibodeau GA. Brief Atlas of the Human Body for Anatomy \& Physiology. 7th edn. USA: St. Louis; 2010. pp. 1224

4. Caryn RR. Regimens: Eat Your Vegetables, but Not Too Many. USA: The New York Times; 2010.p.6

5. Deadman P, Al-Khafaji M, Baker K. A Manual of Acupuncture. $2^{\text {nd }}$ edn. Journal of Chinese Medicine Publications, England. 2007.

6. Shi A. Hypothyroidism, Essentials of Chinese Medicine: Internal Medicine. Bridge Publishing group, USA. 2003.P.66.

7. Bensky D, Clavey S, Stoger E. Chinese Herbal Medicine - Materia Medica. 3rd edn. USA: Eastland Press, Inc., Seattle; 2004.

8. Cheng X. Chinese Acupuncture and Moxibustion, Revised Edition. Foreign Languages Press, Beijing, China. 1999.

9. Scheid V, Bensky D, Ellis A, et al. Chinese Herbal Medicine - Formulas \& Strategies. 2nd edn. USA: Eastland Press, Inc., Seattle; 2009.

10. Cheng D. Acupuncture and Moxibustion Formulas and Treatment. 1st edn. Boulder, USA: Blue Poppy Press, 1996.

11. Huo W, Xu G, Wang H. Treating Autoimmune Disease with Chinese Medicine. USA: Elsevier Ltd; 2011. 